Yipsy Roque Benito

Aplicação de nanofluidos em sistemas secundários de refrigeração

Tese apresentada como requisito parcial para obtenção do grau de Doutor pelo Programa de PósGraduação em Engenharia Mecânica da PUC-Rio.

Orientador: Prof. José Alberto dos Reis Parise Co-orientador: Prof. José Viriato Coelho Vargas

Rio de Janeiro

Maio de 2012 
Yipsy Roque Benito

\section{Aplicação de nanofluidos em sistemas \\ secundários de refrigeração}

Tese apresentada como requisito parcial para obtenção do grau de Doutor pelo Programa de Pós-Graduação em Engenharia Mecânica da PUC-Rio. Aprovada pela Comissão Examinadora abaixo assinada.

\section{Prof. José Alberto dos Reis Parise}

Orientador

Departamento de Engenharia Mecânica - PUC-Rio

Prof. José Viriato Coelho Vargas

Co-Orientador

Departamento de Engenharia Mecânica - UFPR

Prof. Alcir de Faro Orlando

Departamento de Engenharia Mecânica - PUC-Rio

Prof. Carlos Eduardo Leme Nóbrega

Departamento de Engenharia Mecânica - CEFET/RJ

Prof. Carlos Valois Maciel Braga

Departamento de Engenharia Mecânica - PUC-Rio

Prof. Enio Pedone Bandarra Filho

Faculdade de Engenharia Mecânica - UFU

Prof. Manuel Ernani de Carvalho Cruz Departamento de Engenharia Mecânica - UFRJ

Prof. Sergio Leal Braga Departamento de Engenharia Mecânica - PUC-Rio

Prof. José Eugenio Leal Coordenador Setorial do Centro Técnico Científico - PUC-Rio 
Todos os direitos reservados. É proibida a reprodução total ou parcial do trabalho sem autorização da universidade, da autora e do orientador.

\section{Yipsy Roque Benito}

Graduou-se em Engenharia Mecânica na Universidad Central "Marta Abreu" de Las Villas, Cuba, em 1998. Possui Mestrado em Engenharia Mecânica, na área de concentração Petróleo e energia, pela PUC-Rio (2007). Atua na área de Engenharia Mecânica, com ênfase em Refrigeração e Aproveitamento da Energia. Tem trabalhado em análises de sistemas energéticos.

Ficha Catalográfica

Benito, Yipsy Roque

Aplicação de nanofluidos em sistemas secundários de refrigeração / Yipsy Roque Benito; orientadores: José Alberto dos Reis Parise, José Viriato Coelho Vargas. 2012.

172 f. : il. (color.) ; $30 \mathrm{~cm}$

Tese (doutorado)-Pontifícia Universidade Católica do Rio de Janeiro, Departamento de Engenharia Mecânica, 2012.

Inclui bibliografia

Engenharia Mecânica - Teses. 2. Nanofluido. 3. Sistema indireto de refrigeração. 4. Coeficiente estrutural. 5. Análise termoeconômica. I. Parise, José Alberto dos Reis. II. Vargas, José Viriato Coelho. III. Pontifícia Universidade Católica do Rio de Janeiro. Departamento de Engenharia Mecânica. IV. Título. 
Para mi abuela Concha, que acreditaba que doctorado era cosa de Medicina. 


\section{Agradecimentos}

Às agências CNPq e FAPERJ e à PUC-Rio, pelos auxílios concedidos, sem os quais este trabalho não poderia ter sido realizado.

Ao meu orientador, Prof. José Alberto dos Reis Parise, por todos os ensinamentos e pelos desafios colocados, os quais foram, sem dúvidas, incentivos para o crescimento.

Ao meu co-orientador, Prof. José Viriato Coelho Vargas, pelo apoio e estímulo.

Ao Jesús Betancourt Mena, professor, colega e, uma vez mais, tutor alternativo.

Ao meu pai, Pablo Roque Diaz, por me apontar os caminhos na vida e na engenharia.

À minha mãe, Margot Benito Blanco por ser motivo constante de inspiração por sua força e sua beleza.

À professora Irenia Gallardo, pelas oportunas colaborações.

Aos colegas da PUC-Rio: Frank, Paul, Isela, Minchola, Jubs, Jujú, Edwin e Marcos pelos ricos debates e as contribuições inapreciáveis ao presente trabalho.

Aos colegas do Laboratório de Máquinas Hidráulicas, na UFPR, pela colaboração no levantamento dos dados experimentais.

Aos funcionários do Departamento de Engenharia Mecânica da PUC-Rio, pela ajuda em momentos cruciais.

Aos amigos daqui e aos de Santa Clara, em especial a Lisbet, pela presença, pela paciência e pelo carinho.

À Xenia, que por muitas razões não pode estar ausente destes agradecimentos.

Ao Pedro Rocha de Oliveira, companheiro da minha vida, por isso e por aprender direitinho o ciclo de refrigeração. 


\section{Resumo}

Benito, Yipsy Roque; Parise, José Alberto dos Reis. Aplicação de nanofluidos em sistemas secundários de refrigeração. Rio de Janeiro, 2012. 172p. Tese de Doutorado - Departamento de Engenharia Mecânica, Pontifícia Universidade Católica do Rio de Janeiro.

É estudada a aplicação de nanofluidos como fluidos secundários em sistemas de refrigeração por compressão de vapor mediante o desenvolvimento de um modelo termodinâmico de parâmetros concentrados. Quando um nanofluido é usado como fluido térmico, sua condutividade e viscosidade aumentam com respeito às propriedades do fluido base correspondente. Como conseqüência, a irreversibilidade por transferência calor diminui enquanto que a por atrito aumenta. É aplicado o método dos coeficientes estruturais para determinar o efeito da concentração de nanopartículas no fluido secundário na irreversibilidade global do sistema, levando em consideração as inter-relações da estrutura analisada. Para estimar os limites práticos da redução da irreversibilidade térmica com o uso de nanofluidos é proposta uma otimização do custo operacional, a partir de análise termoeconômica, considerando a aplicação do novo fluido secundário no sistema, sem nenhuma outra modificação no mesmo. A partir do modelo proposto, verificado com dados experimentais do ciclo de refrigeração, simulou-se um caso particular de operação. Mediante uma otimização parcial, foi determinado o ponto de mínimo custo operacional, com a simples variação da concentração volumétrica de nanopartículas. Os resultados das otimizações fornecem diferentes valores da concentração ótima para diferentes cenários, caracterizados por vários comprimentos equivalentes do circuito secundário e diversos tempos de operação anual. Adicionalmente, o trabalho inclui um estudo sobre a aplicação de nanofluidos em um evaporador de casco e tubo, o qual foi simulado a partir de um modelo termodinâmico detalhado. Dados experimentais foram levantados para validar o modelo.

\section{Palavras-chave}

Nanofluido; sistema indireto de refrigeração; coeficiente estrutural; análise termoeconômica 


\section{Abstract}

Benito, Yipsy Roque; Parise, José Alberto dos Reis (Advisor). Application of nanofluids in secondary refrigeration systems. Rio de Janeiro, 2012. 172p. Dsc. Thesis - Departamento de Engenharia Mecânica, Pontifícia Universidade Católica do Rio de Janeiro.

The application of nanofluids as secondary fluids in vapor compression refrigeration systems is studied with the development of a lumped-parameter thermodynamic model. When a nanofluid is used as a heat transfer fluid, its thermal conductivity and viscosity increase, when compared with the corresponding properties of the base fluid. The irreversibilities due to heat transfer and due to friction decrease and increase, respectively. After irreversibility is calculated for each component, the method of structural coefficients of internal bonds is applied to determine the effect of the volumetric concentration of nanoparticles in the secondary fluid on the system's global irreversibility, taking into account the interrelations of the analyzed structure. To estimate the practical limits of thermal irreversibility reduction with nanofluid application, an optimization of operational cost was proposed, based on thermoeconomic analysis, and considering the application of the new secondary fluid on the system, without additional modifications. Based on the proposed model, which was verified by experimental data, an typical operation condition was simulated. Through partial optimization, the minimum operational cost is determined for a simple variation of volumetric concentration of nanoparticles. The results of the optimizations furnish different optimal concentration values for different scenarios. Additionally, an study of nanofluid application in a shell and tube evaporator was included. The evaporator was simulated from a detailed thermodynamic model. Experimental data were collected to validate the model.

\section{Keywords}

Nanofluid; indirect vapor compression refrigeration systems; structural coefficent; thermoeconomic analysis 


\section{Sumário}

1. Introdução 22

1.1 Sistemas indiretos e nanofluidos $\quad 22$

1.2 Estado da arte $\quad 24$

1.2.1 Nanofluidos: visão geral 25

1.2.2 Critérios de avaliação $\quad 26$

1.2.3 Aplicações como fluidos térmicos $\quad 27$

1.2.4 Nanofluidos como fluidos secundários em refrigeração 28

$\begin{array}{ll}\text { 1.2.5 Nanofluidos em sistemas } & 29\end{array}$

$\begin{array}{ll}1.3 \text { Justificativa e contextualização } & 29\end{array}$

$\begin{array}{ll}1.4 \text { Metodologia } & 30\end{array}$

1.5 Objetivos da tese 32

1.6 Organização do trabalho 32

2. Mecânica dos fluidos, transferência de calor e termodinâmica 34 aplicadas a nanofluidos

2.1 Modelo de transporte térmico em nanofluidos 34

2.1.1 Propriedades 34

2.1.1.1 Massa específica $\quad 35$

2.1.1.2 Calor específico $\quad 35$

2.1.1.3 Condutividade térmica $\quad 35$

2.1.1.4 Viscosidade dinâmica em nanofluidos $\quad 38$

2.1.2 Números adimensionais $\quad 44$

2.2 Transferência de calor por convecção forçada em nanofluidos 44

2.2.1 Investigações teóricas $\quad 45$

$\begin{array}{ll}\text { 2.2.2 Correlações experimentais } & 47\end{array}$

2.3 Queda de pressão em nanofluidos 49

2.4 Exergia do nanofluido 52

2.4.1 Exergia de uma corrente de nanofluido 52

2.4.1.1 Entalpia e entropia específicas de um nanofluido 53

2.4.1.3 Exergias química e física dos nanofluidos $\quad 55$

2.5 Nanofluidos em trocadores de calor $\quad 56$

3. Modelagem do uso de nanofluidos como fluidos secundários na 58 refrigeração por compressão de vapor

3.1. Descrição do sistema de refrigeração por compressão de vapor 58 
3.2. Ciclo termodinâmico 59

3.3. Sistema indireto de refrigeração 61

3.4. Irreversibilidade 63

3.4.1. Irreversibilidade devido à queda de pressão 64

3.4.2. Irreversibilidade devido uma diferença finita de temperaturas 65

3.4.3. Irreversibilidade total em um trocador de calor 66

3.5. Modelo matemático 68

$\begin{array}{ll}\text { 3.5.1 Hipóteses simplificadoras } & 68\end{array}$

3.5.2 Balanços de energia e exergia dos componentes do ciclo 69

$\begin{array}{ll}3.5 .2 .1 \text { Compressor } & 70\end{array}$

$\begin{array}{ll}\text { 3.5.2.2 Trocadores de calor } & 72\end{array}$

3.5.2.3 Modelo geral da bomba de líquido do circuito secundário 78

$\begin{array}{ll}\text { 3.5.2.4 Modelo geral do dispositivo de expansão } & 78\end{array}$

$\begin{array}{ll}\text { 3.5.2.5 Balanço global de energia } & 79\end{array}$

3.5.2 Análise exergética do sistema indireto de refrigeração 79

3.5.3.1 Balanços exergéticos dos sub-sistemas $\quad 81$

3.5.3.2 Irreversibilidade dos trocadores do circuito secundário 83

3.5 Indicadores térmicos de desempenho global para o sistema indireto de 84 refrigeração

3.5.1 Coeficiente de desempenho: COP 84

3.5.2 Eficiência racional 85

4. Análise termoeconômica 86

4.1. Otimização 86

4.2. Coeficientes estruturais 87

4.3. Procedimento para a otimização termoeconômica pelo método dos 89 coeficientes estruturais

4.4. Otimização termoeconômica de sistemas indiretos de refrigeração com 90 trocadores de calor que usam nanofluidos

$\begin{array}{ll}\text { 4.4.1 Variáveis de decisão } & 90\end{array}$

4.4.2 Irreversibilidade do subsistema k da planta em função da variável de 91 decisão

4.4.3 Variação da taxa de irreversibilidade do trocador secundário com a 92 variação da variável de decisão

4.4.4 Definição da irreversibilidade total da planta em função da variável de 92 decisão e sua derivada

4.4.5 Coeficiente estrutural 93

$\begin{array}{ll}4.4 .6 \text { Custos } & 94\end{array}$

$\begin{array}{ll}5 \text { Simulação } & 97\end{array}$ 
$\begin{array}{ll}\text { 5.1 Modelo matemático } & 97\end{array}$

$\begin{array}{ll}\text { 5.2 Solução numérica } & 97\end{array}$

5.3 Validação do modelo do ciclo termodinâmico com dados experimentais 99

$\begin{array}{ll}5.4 \text { Estudo de caso } & 101\end{array}$

5.4.1 Resultados da simulação 103

5.4.2 Análise econômica 115

5.4.2.1 Custos monetários 116

5.4.2.2 Otimização termoeconômica 118

5.5 Incertezas da simulação e da otimização 123

6 Simulação de um evaporador casco-e-tubo (em posição vertical e com 125 fluido ascendente) esfriando um nanofluido

6.1 Introdução 125

6.2 Modelo 126

$\begin{array}{ll}\text { 6.2.1 Hipóteses simplificadoras } & 127\end{array}$

6.2.2 Equações de balanço e troca de calor $\quad 128$

6.2.3 Determinações do coeficiente de transferência de calor por 129 convecção no interior dos tubos

6.2.3.1 Região bifásica 130

6.2.3.1.1 Modelo para escoamento em ebulição 130

6.2.3.1.2 Modelo para a região após a secagem de parede 131

6.2.3.2 Zona de vapor superaquecido 132

6.2.4 Determinação do coeficiente de transferência de calor por convecção 132 no lado do casco

6.2.5 Queda de pressão pelo lado do casco 133

6.3 Solução numérica 135

6.3.1 Metodologia 135

$\begin{array}{ll}\text { 6.3.2 Simulação } & 137\end{array}$

$\begin{array}{ll}\text { 6.3.3 Dados de entrada } & 138\end{array}$

6.3.4 Resultados 139

6.3.4.1 Comparação com dados experimentais 139

6.3.4.2 Estudo do evaporador operando com nanofluido de $\mathrm{Al}_{2} \mathrm{O}_{3}-\mathrm{H}_{2} \mathrm{O} \quad 143$

$\begin{array}{ll}6.4 \text { Análise de resultados } & 144\end{array}$

$\begin{array}{ll}\text { 7. Conclusões } & 149\end{array}$

$\begin{array}{ll}7.1 \text { Conclusões } & 149\end{array}$

7.2 Recomendações para trabalhos futuros 152

8. Referências bibliográficas 153

$\begin{array}{ll}\text { Apêndice } & 168\end{array}$ 


\section{Lista de figuras}

Figura. 1. Viscosidade efetiva para nanofluido $\mathrm{Al}_{2} \mathrm{O}_{3}$ - água segundo

a correlação de Khanafer e Vafai (2011) $1 \% \leq \varphi_{n p} \leq 9 \%$;

$20^{\circ} \mathrm{C} \leq T \leq 70^{\circ} \mathrm{C} ; \quad 13 \mathrm{~nm} \leq d_{n p} \leq 131 \mathrm{~nm}$

Figura 2. Representação esquemática de um sistema de 58 refrigeração por compressão

Figura 3. Ciclo básico ideal de refrigeração

Figura 4. Ciclo real de refrigeração, considerando graus de subresfriamento e superaquecimento, queda de pressão nos trocadores de calor e compressão politrópica

Figura 5. Esquema do sistema indireto de refrigeração

Figura 6. Distribuição de temperaturas no circuito secundário

Figura 7. Plano T-s de um processo de troca de calor

Figura 8. Esquema do sistema indireto de refrigeração

Figura 9. Fluxos de energia no sistema indireto de refrigeração

Figura 10. Volume de controle do sistema indireto de refrigeração por compressão de vapor

Figura 11. Fluxos de exergia no sistema indireto de refrigeração

Figura 12. Comparação do desempenho experimental e calculado 100 para o ciclo de amônia

Figura 13. Efeito da concentração volumétrica de nanopartículas no 104 desempenho do ciclo e no desempenho global. Para $\mathrm{Re}=13000 \mathrm{e}$ um comprimento do circuito secundário de $10 \mathrm{~m}$

Figura 14. Efeito da concentração volumétrica de nanopartículas no 105 trabalho de compressão. Para $\mathrm{Re}=13000$

Figura 15. Efeito da concentração volumétrica de nanopartículas na 106 pressão de evaporação. Para $\operatorname{Re}=13000$ 
Figura 16. Efeito da concentração volumétrica de nanopartículas no 106 trabalho de compressão e de bombeamento para três comprimentos equivalentes deferentes: $L 1=0 \mathrm{~m} ; \mathrm{L} 2=10 \mathrm{~m} ; \mathrm{L} 3=100$

Figura 17. Efeito da concentração volumétrica de nanopartículas na 107 eficiência racional global. Para $R e=13000$ e um comprimento do circuito secundário de $10 \mathrm{~m}$

Figura 18. Efeito da concentração volumétrica de nanopartículas na 108 eficiência racional global. Para $\mathrm{Re}=13000$ e para três comprimentos equivalentes deferentes: $L 1=0 \mathrm{~m} ; \mathrm{L} 2=10 \mathrm{~m} ; \mathrm{L} 3=100$

Figura 19. Irreversibilidades dos componentes do sistema variando a 108 concentração volumétrica de nanopartículas. Para $R e=13000$ e um comprimento do circuito secundário de $10 \mathrm{~m}$

Figura 20. Irreversibilidades dos componentes do circuito secundário 109 variando a concentração volumétrica de nanopartículas. Para $\mathrm{Re}=$ 13000 e um comprimento do circuito secundário de $10 \mathrm{~m}$

Figura 21. Irreversibilidades do trocador secundário variando a 109 concentração volumétrica de nanopartículas. Para $R e=13000$

Figura 22. Efeito da concentração volumétrica de nanopartículas na 110 diferença de temperaturas média logarítmica no evaporador e no trocador secundário. Para $\mathrm{Re}=13000$

Figura 23. Vazão de fluido secundário em função da concentração 111 volumétrica de nanopartículas, para $R e=13000$

Figura 24. Coeficiente estrutural para o trocador secundário. L1=0m; 112 $\mathrm{L} 2=10 \mathrm{~m} ; \mathrm{L} 3=100$

Figura 25. Variação do coeficiente estrutural do trocador secundário 113 com taxa de irreversibilidade do trocador secundário. Comprimento das tubulações do circuito secundário $100 \mathrm{~m}$

Figura 26. Variação do coeficiente estrutural do trocador secundário 114 com a concentração volumétrica de nanopartículas. Comprimento das tubulações do circuito secundário 100m 
Figura 27. Variação das irreversibilidades do trocador secundário e 114 da bomba com a concentração volumétrica de nanopartículas. Comprimento das tubulações do circuito secundário $100 \mathrm{~m}$

Figura 28. Componentes do custo para um tempo de operação de 117 $8760 \mathrm{~h} / \mathrm{ano}$, comprimento equivalente do circuito secundário de $10 \mathrm{~m}$ Figura 29. Custo de operação para um período de operação de 8760118 $\mathrm{h} /$ ano, comprimento comprimentos $L 1=0, L 2=10 \mathrm{~m}$ e $L 3=100 \mathrm{~m}$

Figura 30a. Otimização gráfica para um tempo de operação de 8760119 h/dia, comprimentos L1 e L2

Figura 30b. Otimização gráfica para um tempo de operação de 8760120 h/ano, comprimento L3

Figura 31a. Otimização gráfica para um comprimento desprezível 121 das tubulações do circuito secundário

Figura 31b. Otimização gráfica para um comprimento das tubulações 121 do circuito secundário igual a $10 \mathrm{~m}$

Figura 31c. Otimização gráfica para um comprimento das tubulações 122 do circuito secundário igual a $100 \mathrm{~m}$

Figura 32. Representação esquemática do evaporador 126

Figura 33. Esquema do elemento "i” de trocador

Figura 34a. Diagrama de blocos contendo o algoritmo básico de 136 solução do programa principal

Figura 34b. Diagrama de blocos contendo o algoritmo da sub-rotina 137 de correção da temperatura de saída do fluido refrigerado

Figura 35. Teste de malha para a taxa de transferência de calor

Figura 36. Protótipo do refrigerador de absorção da UFPR 140

Figura 37. Vista parcial do evaporador na instalação da UFPR $\quad 140$

Figura 38. Taxa de transferência de calor no evaporador. 142 Comparação entre resultados do programa e medições experimentais para água 
Figura 39. Perfil de temperaturas para o evaporador operando com 143 água. Cem divisões

Figura 40. Incremento da condutividade com respeito ao fluido base 145 para Re constante e igual a 2100. $d_{p}=20 \mathrm{~nm}$

Figura 41. Incremento da viscosidade dinâmica com respeito ao 145 fluido para Re constante e igual a 2100. $d_{p}=20 \mathrm{~nm}$

Figura 42a. Fatores de incremento da condutividade, o coeficiente 146 de transferência de calor e a taxa de transferência de calor para capacidade calorífica constante

Figura 42b. Fatores de incremento da condutividade, o coeficiente 146 de transferência de calor e a taxa de transferência de calor para capacidade volumétrica constante

Figura 42c. Fatores de incremento da condutividade, o coeficiente de 147 transferência de calor e a taxa de transferência de calor para $\mathrm{Re}$ constante. $d_{p}=20 \mathrm{~nm}$

Figura 43. Fatores de incremento da queda de pressão pelo lado do 148 fluido refrigerado para diferentes critérios. $d_{p}=20 \mathrm{~nm}$ 


\section{Lista de tabelas}

Tabela 1. Correlações experimentais de convecção forçada 47 (adaptada de Sarkar, 2011)

Tabela 2. Equações de balanços e irreversibilidades para os 98 componentes do sistema

Tabela 3. Dados experimentais (Bingming et al, 2009) 100

Tabela 4. Dados de entrada para o estudo de caso 101

Tabela 5. Resultados numéricos para o sistema base operando com 103 água como fluido secundário, para um comprimento do circuito secundário de $10 \mathrm{~m}$

Tabela 6. Coeficientes estruturais para os componentes do circuito 111 secundário. Comprimento equivalente do circuito secundário igual a $10 \mathrm{~m}$

Tabela 7. Concentração ótima para mínimo custo de operação anual 123

Tabela 8. Incertezas dos instrumentos de medição 


\section{Simbologia}

\begin{tabular}{|c|c|c|}
\hline$A$ & Área & $\mathrm{m}^{2}$ \\
\hline$A_{L}$ & Área de transferência de calor & $\mathrm{m}^{2}$ \\
\hline$A_{T}$ & Área de escoamento & $\mathrm{m}^{2}$ \\
\hline$B C$ & Corte do defletor & $\%$ \\
\hline$c$ & Concentração mássica da mistura & - \\
\hline C & Capacidade térmica & $\mathrm{kW} / \mathrm{K}$ \\
\hline$C$ & Custo & $\$$ \\
\hline COP & Coeficiente de desempenho & - \\
\hline$c_{P}$ & Calor específico a pressão constante & $\mathrm{kJ} /(\mathrm{kg} \mathrm{K})$ \\
\hline$d_{P}$ & Diâmetro de partícula & $\mathrm{nm}$ \\
\hline$D b_{\text {inlet }}$ & Diâmetro bocal de entrada & $\mathrm{m}$ \\
\hline$D b_{\text {outet }}$ & Diâmetro bocal de saída & $\mathrm{m}$ \\
\hline$D_{h}$ & Diâmetro hidráulico & $\mathrm{m}$ \\
\hline Dotl & $\begin{array}{l}\text { Diâmetro do circulo formado pelos diâmetros exteriores } \\
\text { dos tubos }\end{array}$ & $\mathrm{m}$ \\
\hline$D t$ & Diâmetro de tubo & $\mathrm{m}$ \\
\hline$D s_{e x t}$ & Diâmetro exterior do casco & $\mathrm{m}$ \\
\hline$e$ & Exergia específica & $\mathrm{kJ} / \mathrm{kg}$ \\
\hline$\dot{E}$ & Taxa de exergia & $\mathrm{kW}$ \\
\hline$\dot{E}_{C}^{Q}$ & $\begin{array}{l}\text { Taxa de exergia de entrada ao sistema indireto de } \\
\text { refrigeração }\end{array}$ & $\mathrm{kW}$ \\
\hline$f$ & Fator de atrito & - \\
\hline$F_{R \$ / U S D}$ & Taxa de câmbio vigente entre moedas & \\
\hline$g$ & Aceleração gravitacional & $\mathrm{m} / \mathrm{s}^{2}$ \\
\hline$G$ & Velocidade mássica & $\mathrm{kg} /\left(\mathrm{s} \mathrm{m}^{2}\right)$ \\
\hline$h$ & Entalpia específica & $\mathrm{kJ} / \mathrm{kg}$ \\
\hline$\dot{I}$ & Taxa de irreversibilidade & $\mathrm{kW}$ \\
\hline$\dot{I}_{P}$ & Taxa de irreversibilidade mecânica ou devido à queda de & $\mathrm{kW}$ \\
\hline
\end{tabular}




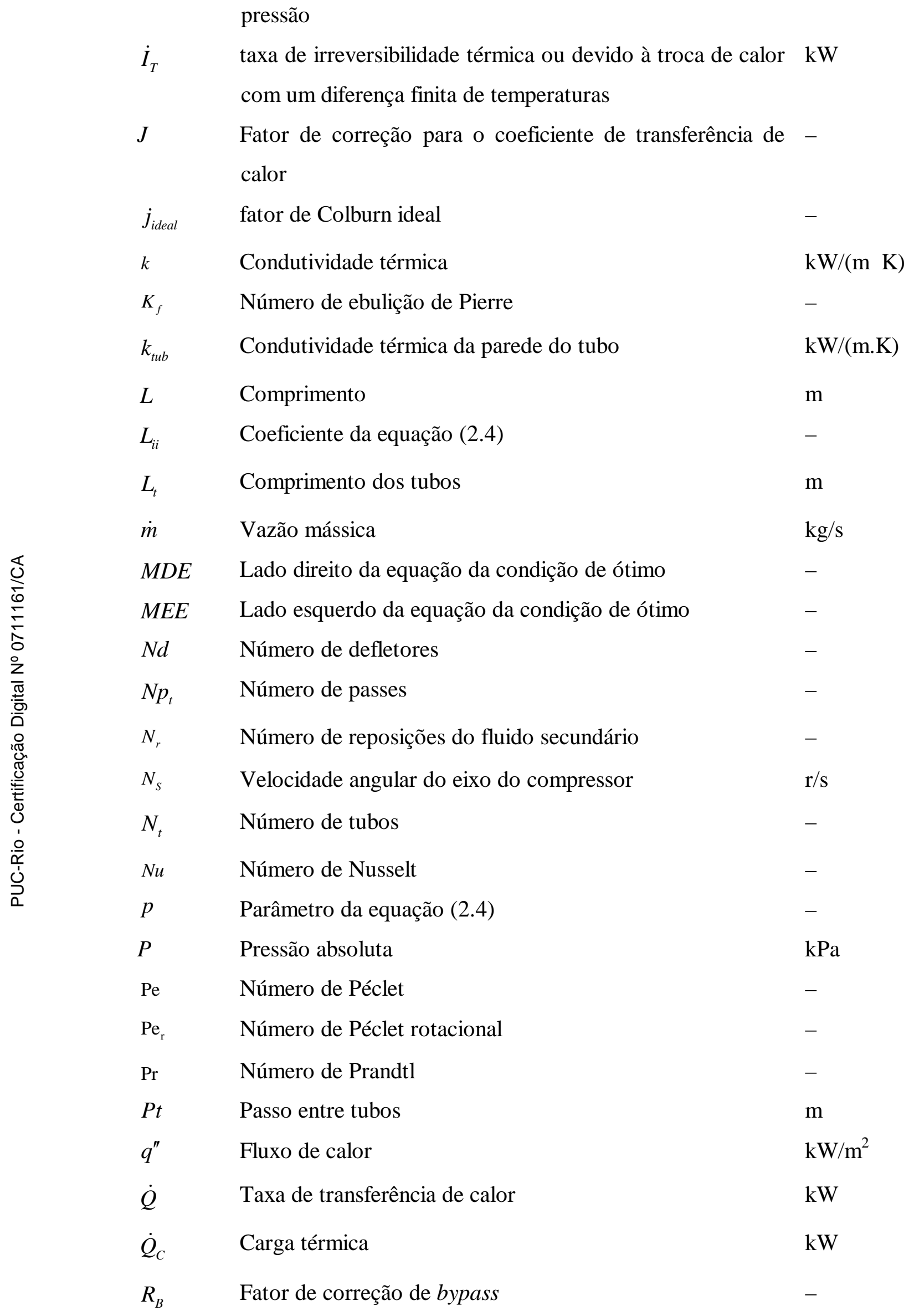




\begin{tabular}{|c|c|c|}
\hline$R_{b d}$ & Resistência interfacial térmica de Kapitza & - \\
\hline$R_{f}$ & Resistência térmica devido à incrustação & $\mathrm{m}^{2} \mathrm{~K} / \mathrm{kW}$ \\
\hline$R_{k}$ & Incremento da condutividade & - \\
\hline$R_{L}$ & Fator de correção de fugas & - \\
\hline$R_{\dot{Q}}$ & Incremento da taxa de transferência de calor & - \\
\hline$R_{\mu}$ & Incremento da viscosidade & - \\
\hline$R_{\alpha}$ & Incremento do coeficiente de transferência de calor & - \\
\hline$R_{\Delta P}$ & $\begin{array}{l}\text { Incremento da queda de pressão pelo lado do fluido } \\
\text { resfriado }\end{array}$ & - \\
\hline $\operatorname{Re}$ & Número de Reynolds & - \\
\hline$r_{\text {comp }}$ & razão de compressão & - \\
\hline$s$ & Entropia específica & $\mathrm{kJ} /(\mathrm{kgK})$ \\
\hline$\dot{S}_{g e n}$ & Taxa de geração de entropia & $\mathrm{kW} / \mathrm{K}$ \\
\hline$t$ & Tempo & $\mathrm{s}$ \\
\hline$t_{o p}$ & Tempo de operação anual & $\mathrm{h} / \mathrm{ano}$ \\
\hline$T$ & Temperatura absoluta & $\mathrm{K}$ \\
\hline $\bar{T}$ & Temperatura média & $\mathrm{K}$ \\
\hline$U$ & Coeficiente global de transferência de calor & $\mathrm{kW} /\left(\mathrm{m}^{2} \mathrm{~K}\right)$ \\
\hline$u_{m}$ & Velocidade média & $\mathrm{m} / \mathrm{s}$ \\
\hline$V$ & Velocidade & $\mathrm{m} / \mathrm{s}$ \\
\hline$\dot{V}$ & Vazão volumétrica & $\mathrm{m}^{3} / \mathrm{s}$ \\
\hline$v$ & Volume específico & $\mathrm{m}^{3} / \mathrm{kg}$ \\
\hline$\forall_{n f}$ & Inventário volumétrico anual de nanofluido & $\mathrm{m}^{3}$ \\
\hline$\dot{W}$ & Taxa de trabalho fornecido ou potência elétrica & $\mathrm{kW}$ \\
\hline$x$ & Fração mássica de vapor ou título & - \\
\hline
\end{tabular}




\section{Símbolos gregos}

\begin{tabular}{|c|c|c|}
\hline$\alpha$ & Coeficiente de transferência de calor & $\mathrm{kW} /\left(\mathrm{m}^{2} \mathrm{~K}\right)$ \\
\hline$\alpha_{\mathrm{tp}}$ & $\begin{array}{l}\text { Coeficiente local de troca de calor para ebulição no } \\
\text { interior dos tubos }\end{array}$ & $\mathrm{kW} /\left(\mathrm{m}^{2} \mathrm{~K}\right)$ \\
\hline$\beta_{i i}$ & Coeficiente da equação (2.4) & - \\
\hline$\gamma$ & Parâmetro da equação (2.4) & - \\
\hline$\gamma$ & Potencial químico & - \\
\hline$\delta$ & Incerteza & $\mathrm{m}$ \\
\hline$\delta_{t}$ & Espessura do tubo & $\mathrm{m}$ \\
\hline$\delta_{s}$ & Espessura do casco & $\mathrm{m}$ \\
\hline$\delta_{n f}$ & Difusividade térmica do nanofluido & $\mathrm{m}^{2} / \mathrm{s}$ \\
\hline$\delta_{v}^{+}$ & Espessura adimensional da sub-camada laminar & - \\
\hline$\Delta$ & Variação & - \\
\hline$\varepsilon$ & Efetividade & - \\
\hline$\zeta$ & Rugosidade da tubulação & $\mathrm{m}$ \\
\hline$\zeta_{\Delta T}$ & Margem de erro para o cálculo da temperatura & - \\
\hline$\theta$ & Arranjo dos tubos no feixe & $\circ$ \\
\hline$\eta$ & Eficiência & - \\
\hline$\kappa$ & Constante de proporcionalidade & - \\
\hline$k_{i i}^{c}$ & Parâmetro da equação (2.4) & - \\
\hline$\mu$ & Viscosidade & - \\
\hline$\rho$ & Massa específica & $\mathrm{kg} / \mathrm{m}^{3}$ \\
\hline$\varphi$ & Concentração volumétrica de partículas & - \\
\hline$\sigma$ & Coeficiente estrutural & - \\
\hline$\psi$ & Eficiência racional & - \\
\hline
\end{tabular}




\section{Sub-índices e abreviaturas}

\begin{tabular}{|c|c|}
\hline 0 & Meio ambiente \\
\hline app & Aproximação \\
\hline$b$ & Componente do custo independe da variável de decisão \\
\hline$b f$ & Fluido base \\
\hline bundle & Feixe de tubos \\
\hline$c$ & Corrente fria no trocador de calor \\
\hline calc & Calculado \\
\hline$c h$ & Química \\
\hline ciclo & Referente ao ciclo de refrigeração \\
\hline cnd & Condensação \\
\hline crit & Crítico \\
\hline CON & Condensador \\
\hline des & Dessuperaquecimento \\
\hline eff & Efetivo \\
\hline el & Eletricidade \\
\hline $\exp$ & Experimental \\
\hline ext & exterior \\
\hline$E V A$ & Evaporador \\
\hline$f$ & fluido \\
\hline$f w$ & Fluido refrigerado \\
\hline$h$ & Corrente quente no trocador de calor \\
\hline$H E$ & Trocador de calor \\
\hline$i$ & Referente a um elemento de trocador \\
\hline ideal & Ideal \\
\hline in & Entrada ao volume de controle \\
\hline int & Interno \\
\hline$k$ & cinética \\
\hline$k$ & Referente ao sub-sistema k-ésimo \\
\hline$l$ & Líquido \\
\hline $\operatorname{lm}$ & Media logarítmica \\
\hline
\end{tabular}




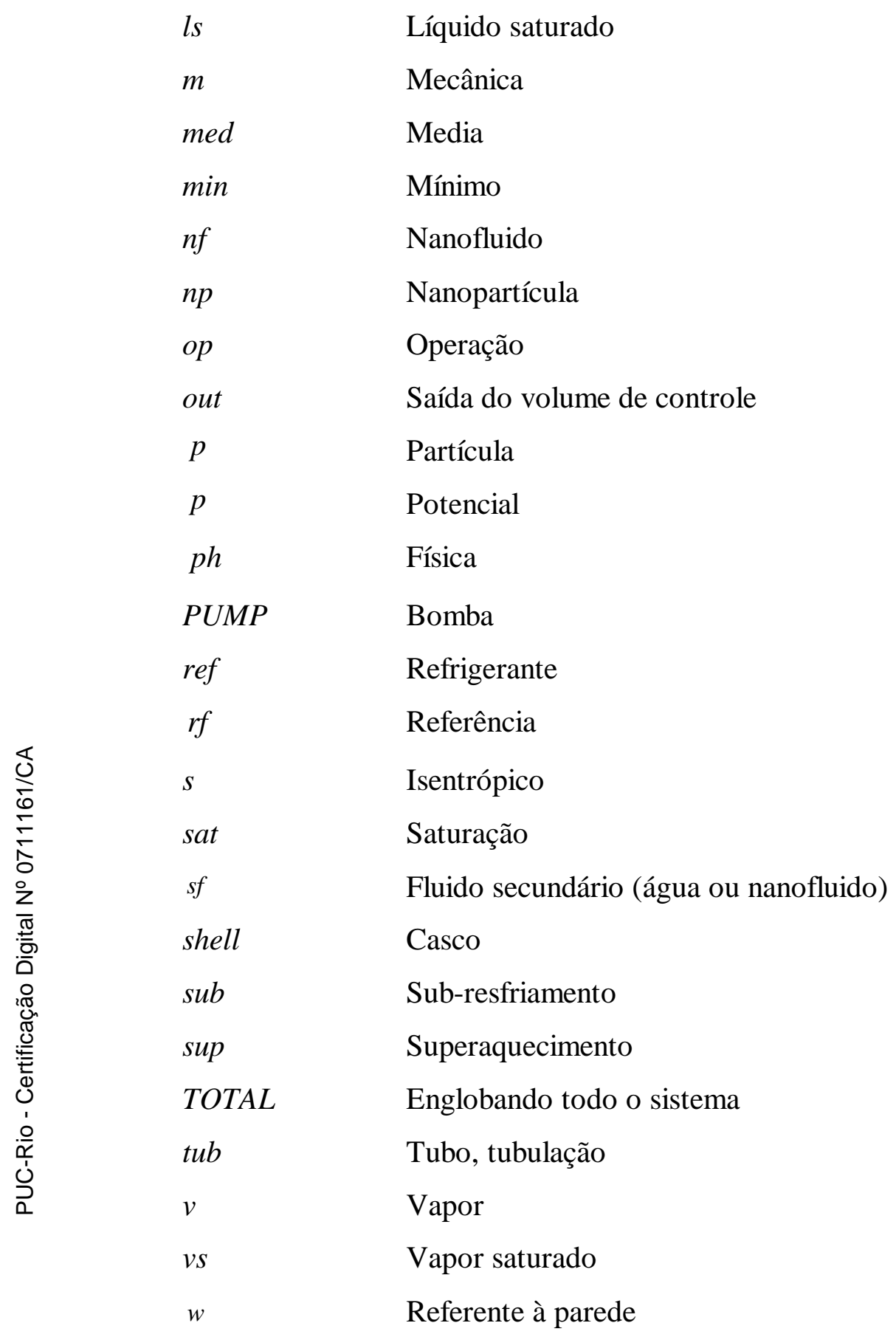

